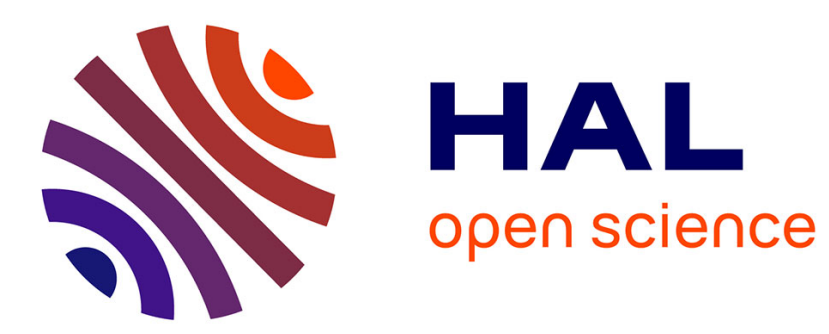

\title{
Analysis of the modified mass method for the dynamic Signorini problem with Coulomb friction
}

David Doyen, Alexandre Ern

\section{To cite this version:}

David Doyen, Alexandre Ern. Analysis of the modified mass method for the dynamic Signorini problem with Coulomb friction. SIAM Journal on Numerical Analysis, 2011, 49 (5), pp.2039-2056. 10.1137/100804711. hal-00473809

\section{HAL Id: hal-00473809 \\ https://hal.science/hal-00473809}

Submitted on 16 Apr 2010

HAL is a multi-disciplinary open access archive for the deposit and dissemination of scientific research documents, whether they are published or not. The documents may come from teaching and research institutions in France or abroad, or from public or private research centers.
L'archive ouverte pluridisciplinaire HAL, est destinée au dépôt et à la diffusion de documents scientifiques de niveau recherche, publiés ou non, émanant des établissements d'enseignement et de recherche français ou étrangers, des laboratoires publics ou privés. 


\title{
Analysis of the modified mass method for the dynamic Signorini problem with Coulomb friction
}

\author{
David Doyen · Alexandre Ern
}

Received: date / Accepted: date

\begin{abstract}
The aim of the present work is to analyze the modified mass method for the dynamic Signorini problem with Coulomb friction. We prove that the space semidiscrete problem is equivalent to an upper semi-continuous one-sided Lipschitz differential inclusion and is, therefore, well-posed. We derive an energy balance. Next, considering an implicit time-integration scheme, we prove that, under a certain condition on the discretization parameters, the fully discrete problem is well-posed. For a fixed discretization in space, we prove also that the fully discrete solutions converge to the space semi-discrete solution when the time step tends to zero.
\end{abstract}

Keywords Finite elements · Time-integration scheme - Elastodynamics · Unilateral contact · Coulomb friction · Differential inclusion · Modified mass method

Mathematics Subject Classification (2000) 65M12 - 65M60 - 74H15 - 74M10 . $74 \mathrm{M} 15 \cdot 74 \mathrm{~S} 05$

\section{Introduction}

The modified mass method is a new approach for solving computationally dynamic problems with unilateral contact. Introduced in [16] for frictionless contact problems, it is based on a space semi-discrete formulation in which the mass matrix is modified (the entries of the mass matrix associated with the (normal) displacements at the contact boundary are set to zero). This modified semi-discrete problem can then be discretized with various time-integration schemes, either implicit or semi-explicit. In the implicit case, the modified mass method enables to eliminate the large spurious oscillations on the contact pressure, which can appear with a standard mass matrix,

D. Doyen

EDF R\&D, 1 avenue du Général de Gaulle, 92141 Clamart Cedex, France

E-mail: david.doyen@edf.fr

A. Ern

Université Paris-Est, CERMICS, Ecole des Ponts, 77455 Marne la Vallée Cedex 2, France

E-mail: ern@cermics.enpc.fr 
while ensuring an exact enforcement of the contact condition. The method does not require extra steps or extra parameters and can easily be implemented. With a suitable scheme such as the Newmark scheme (trapezoidal rule), the modified mass method achieves a tight energy conservation and exhibits a good behavior in long-time. The method can also be used in the context of a semi-explicit time-marching method, with in practice the same stability condition as in the linear case while ensuring an exact enforcement of the contact condition. For a detailed presentation of the modified mass method for frictionless contact and a comparison with other methods, we refer to [8].

The modified mass method has been formulated for dynamic contact problems with Coulomb friction in $[15,11,12]$ and numerical simulations have been performed in $[11,12]$. Up to date, no theoretical analysis has been carried out for the modified mass method in the frictional case. In the frictionless case with an elastic material, interesting results have been proven. The space semi-discrete problem is equivalent to a Lipschitz system of ordinary differential equations and is, therefore, well-posed [16]. The variation of energy is equal to the work of the external forces; the contact forces do not work [16]. Furthermore, convergence of the semi-discrete solutions to a continuous solution is proven for viscoelastic materials in [7].

The aim of the present work is to analyze the modified mass method for the dynamic Signorini problem with Coulomb friction. We prove that the space semi-discrete problem is equivalent to an upper semi-continuous one-sided Lipschitz differential inclusion $[6,20]$ and is, therefore, well-posed (Theorem 2). Furthermore, the variation of energy is equal to the work of the external forces and friction forces (Theorem 3). For the time discretization, we propose an implicit scheme. Each time step requires solving a nonlinear problem similar to a static friction problem. It is well-known that such a problem can have several solutions [14]. Here we prove that, under a certain condition on the discretization parameters of CFL-type, the fully discrete problem is well-posed (Theorem 4). For a fixed discretization in space, we prove also that the fully discrete solutions converge to the space semi-discrete solution when the time step tends to zero (Theorem 5).

With a standard mass term, proving the existence of a semi-discrete solution to a dynamic contact problem is quite delicate. It is necessary to add an impact law and to work with BV and measures spaces [3,21,2]. The modification of the mass term greatly simplifies the analysis. Indeed, the unilateral contact condition can be eliminated and replaced by a Lipschitz continuous term in the momentum equation [16]. Static and quasi-static Coulomb friction problems can have several solutions [14]. Uniqueness is only obtained for small friction coefficients (see [17, Theorem 11.4] for the static case and [13, Theorem 7.2.1] for the quasi-static case). It is worthwhile to notice that in the dynamic case, uniqueness is recovered. We do not examine the convergence of the discrete solutions to a solution of the continuous problem. Nevertheless, it seems possible to extend the convergence result in [7] (see above) to the case of a non-local Coulomb friction (the non-local Coulomb friction is a regularization of Coulomb friction $[17,5]$ ).

This paper is organized as follows. In Section 2, we formulate the continuous problem. Sections 3 and 4 are devoted to the space semi-discrete and fully discrete problems, respectively. In Section 5, we examine the convergence of the fully discrete solutions 
to the space semi-discrete solution.

\section{Continuous problem}

We consider the infinitesimal deformations of a body occupying a reference domain $\Omega \subset \mathbb{R}^{d}(d=2$ or $d=3)$ during a time interval $[0, T]$. Let $\nu$ be the outward unit normal to $\Omega$. The elasticity tensor is denoted by $\mathcal{A}$ and the mass density by $\rho$. An external load $f$ is applied to the body. Let $u:(0, T) \times \Omega \rightarrow \mathbb{R}^{d}, \epsilon(u):(0, T) \times \Omega \rightarrow \mathbb{R}^{d, d}$, and $\sigma(u):(0, T) \times \Omega \rightarrow \mathbb{R}^{d, d}$ be the displacement field, the linearized strain tensor, and the stress tensor, respectively. Denoting time-derivatives by dots, the momentum conservation equation reads

$$
\rho \ddot{u}-\operatorname{div} \sigma=f, \quad \sigma=\mathcal{A}: \epsilon, \quad \epsilon=\frac{1}{2}\left(\nabla u+{ }^{\mathrm{T}} \nabla u\right) \quad \text { in } \Omega \times(0, T) .
$$

The boundary $\partial \Omega$ is partitioned into three disjoint open subsets $\Gamma^{D}, \Gamma^{N}$, and $\Gamma^{\mathrm{c}}$. Dirichlet and Neumann conditions are prescribed on $\Gamma^{D}$ and $\Gamma^{N}$, respectively,

$$
u=u_{D} \quad \text { on } \Gamma^{D} \times(0, T), \quad \sigma \cdot \nu=f_{N} \quad \text { on } \Gamma^{N} \times(0, T) .
$$

In what follows, we assume $f \in W^{1, \infty}\left(0, T ; L^{2}(\Omega)^{d}\right)$ and $f_{N} \in W^{1, \infty}\left(0, T ; L^{2}\left(\Gamma^{N}\right)^{d}\right)$.

We let $u_{\nu}:=u_{\mid \partial \Omega} \cdot \nu$ and $u_{\tau}:=u_{\mid \partial \Omega}-u_{\nu} \nu$ the normal and tangential displacements on $\partial \Omega$, respectively. We also let $\sigma_{\nu}(u):=\nu \cdot \sigma(u)_{\mid \partial \Omega} \cdot \nu$ and $\sigma_{\tau}(u):=\sigma(u)_{\mid \partial \Omega} \cdot \nu-\sigma(u)_{\nu} \nu$ be the normal and tangential stress on $\partial \Omega$, respectively. Note that $u_{\nu}$ and $\sigma_{\nu}(u)$ are scalars while $u_{\tau}$ and $\sigma_{\tau}(u)$ are vectors in $\mathbb{R}^{d}$. Let $|\cdot|$ denote the Euclidean norm in $\mathbb{R}^{m}, m \geq 1$. On $\Gamma^{\mathrm{c}}$, a unilateral contact condition, also called Signorini condition, and a Coulomb friction (see Fig. 1) are enforced

$$
\begin{array}{ll}
u_{\nu} \leq g, \sigma_{\nu}(u) \leq 0, \sigma_{\nu}(u)\left(u_{\nu}-g\right)=0 & \text { on } \Gamma^{\mathrm{c}} \times(0, T), \\
\left|\sigma_{\tau}(u)\right| \leq \mu\left|\sigma_{\nu}(u)\right| & \text { on } \Gamma^{\mathrm{c}} \times(0, T), \\
\sigma_{\tau}(u)=-\mu \sigma_{\nu}(u) \frac{\dot{u}_{\tau}}{\left|\dot{u}_{\tau}\right|} \quad \text { if } \dot{u}_{\tau} \neq 0 & \text { on } \Gamma^{\mathrm{c}} \times(0, T),
\end{array}
$$

where $\mu$ is the friction coefficient and $g$ is the initial gap. At the initial time, the displacement and velocity fields are prescribed,

$$
u(0)=u^{0}, \quad \dot{u}(0)=v^{0} \quad \text { in } \Omega
$$

The mathematical analysis of the above time-dependent problem entails substantial difficulties. The existence of a weak solution is only proven for a viscoelastic material and a non-local Coulomb friction law [5]. 


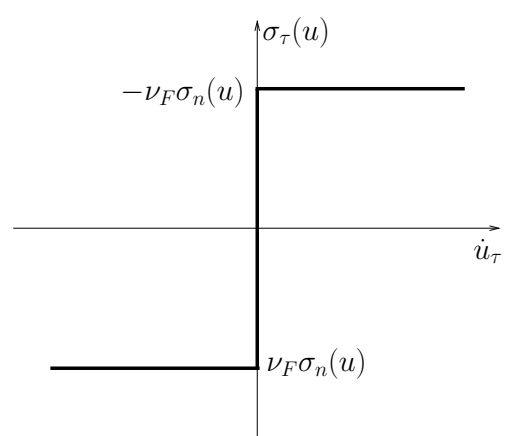

Fig. 1 Coulomb condition $(d=2)$.

\section{Space semi-discrete formulation}

In this section, we formulate the space semi-discrete problem in space and we prove existence and uniqueness of a solution. We also establish an energy balance. In the frictionless case, the semi-discrete problem is equivalent to a Lipschitz ordinary differential equation, and existence and uniqueness are deduced from the Cauchy-Lipschitz theorem. With friction, the situation is more complicated. We choose to model the friction term as a set-valued map. The semi-discrete problem is then equivalent to a differential inclusion, for which generalizations of the Cauchy-Lipschitz theorem are available $[6,20]$.

\subsection{Preliminaries}

To begin with, we introduce some notions needed for the formulation of our problem as a differential inclusion.

- Given a set $E$, we define $\mathcal{P}(E)$ as the set of all subsets of $E$, and $\mathcal{P}^{*}(E):=\mathcal{P}(E) \backslash$ $\{\emptyset\}$.

- A set-valued map is said to be closed convex if its images are closed convex sets.

- Various notions of continuity can be defined for set-valued maps. One of them is upper semi-continuity ${ }^{1}$. A set-valued map $F$ is said to be upper semi-continuous at $x$ if, for every open set $V$ containing $F(x)$, there exists a neighborhood $U$ of $x$ such that $F(U) \subset V$. Consider, for instance, the following set-valued maps:

$$
F_{1}(x)=\left\{\begin{array}{ll}
{[-1,1]} & \text { if } x=0, \\
\{0\} & \text { if } x \neq 0,
\end{array} \text { and } \quad F_{2}(x)= \begin{cases}\{0\} & \text { if } x=0 \\
{[-1,1]} & \text { if } x \neq 0\end{cases}\right.
$$

It is easy to verify that $F_{1}$ is upper semi-continuous for all $x \in \mathbb{R}$, whereas $F_{2}$ is not upper semi-continuous at $x=0$. Here is another example of set-valued-map,

1 This notion of upper semi-continuity is distinct from the upper semi-continuity for singlevalued functions. 
closely related to the Coulomb friction term,

$$
F_{3}(x, y)= \begin{cases}\{-|x|\} & \text { if } y<0 \\ {[-|x|,|x|]} & \text { if } y=0, \\ \{|x|\} & \text { if } y>0\end{cases}
$$

It is easy to verify that this map is upper semi-continuous for all $(x, y) \in \mathbb{R}^{2}$. Finally, we observe that upper semi-continuity applied to single-valued functions is equivalent to continuity. For more details on set-valued maps, we refer to [1].

- The existence theorem for differential inclusions we use does not provide continuously differentiable solutions in time. The solutions are only absolutely continuous in time. For brevity, we do not define this concept and refer to [19]. For our purpose, it suffices to know that an absolutely continuous function $y$ is continuous, differentiable almost everywhere and is equal to the integral of its derivative:

$$
y\left(t_{0}\right)=y(0)+\int_{0}^{t_{0}} \dot{y}(t) d t
$$

Lipschitz continuous functions are absolutely continuous. In what follows, we denote by $A C\left([0, T] ; \mathbb{R}^{m}\right)$, the space spanned by absolutely continuous functions from $[0, T]$ to $\mathbb{R}^{m}$.

- The set-valued maps which appear in our space semi-discrete problem are subgradients and for completeness, we define this notion. Let $J: \mathbb{R}^{m} \rightarrow \mathbb{R} \cup\{+\infty\}$ be a convex function and $D(J):=\left\{v \in \mathbb{R}^{m} ; J(v)<+\infty\right\}$ its domain. We define the subgradient of $J$ as the set-valued map $\partial J: D(J) \rightarrow \mathcal{P}^{*}\left(\mathbb{R}^{m}\right)$ such that

$$
\forall v \in D(J), \quad \partial J(v):=\left\{\gamma \in \mathbb{R}^{m} ; J(w)-J(v) \geq(\gamma, w-v), \forall w \in D(J)\right\},
$$

where $(\cdot, \cdot)$ denotes the canonical inner product on $\mathbb{R}^{m}$. It is easy to prove that the subgradient of a convex function is well-defined and is a closed convex set-valued map. For more details on subgradients, we refer to [4].

We can now state the main result we use for asserting the well-posedness of a problem posed in the form of a differential inclusion.

Theorem 1 Let $P:[0, T] \times \mathbb{R}^{m} \rightarrow \mathcal{P}^{*}\left(\mathbb{R}^{m}\right)$ be a closed convex set-valued map. Let $x_{0} \in \mathbb{R}^{m}$ and consider the following problem: Find $x \in A C\left([0, T] ; \mathbb{R}^{m}\right)$ such that

$$
\begin{aligned}
& \dot{x}(t) \in P(t, x(t)), \\
& x(0)=x_{0} .
\end{aligned}
$$

\section{Assume that}

1. the set-valued map $P(t, \cdot)$ is upper semi-continuous for almost all $t \in[0, T]$;

2. for any $x \in \mathbb{R}^{m}$, there exists a measurable function $p(\cdot, x)$ satisfying $p(t, x) \in P(t, x)$ for almost all $t \in[0, T]$;

3. there exists a function $b \in L^{1}\left(0, T ; \mathbb{R}^{m}\right)$ such that $|p(t, x)| \leq b(t)$ for almost all $t \in[0, T]$. 
Then, there exists a solution to (8)-(9). Furthermore, assume the following one-sided Lipschitz condition: there exists $K \in \mathbb{R}$ such that, for all $t \in[0, T]$, for all $x_{1}, x_{2} \in \mathbb{R}^{m}$,

$$
\left(y_{1}-y_{2}, x_{1}-x_{2}\right) \leq K\left\|x_{1}-x_{2}\right\|^{2}, \quad \forall y_{1} \in P\left(t, x_{1}\right), \forall y_{2} \in P\left(t, x_{2}\right) .
$$

Then, the solution is unique.

Proof For the existence, see [20, Theorem 4.7] or [6, Theorem 5.2]. Uniqueness is straightforward owing to the one-sided Lipschitz condition since it implies that two solutions $x_{1}$ and $x_{2}$ satisfy $\frac{1}{2} \frac{d}{d t}\left(\left\|x_{1}-x_{2}\right\|^{2}\right) \leq K\left\|x_{1}-x_{2}\right\|^{2}$.

Remark 1 In the single-valued case $\left(P:[0, T] \times \mathbb{R}^{m} \rightarrow \mathbb{R}^{m}\right)$, the hypotheses of Theorem 1 become

1. $P(t, \cdot)$ is continuous for almost all $t \in[0, T]$;

2. for any $x \in \mathbb{R}, P(\cdot, x)$ is measurable;

3. there exists a function $b \in L^{1}(0, T ; \mathbb{R})$ such that $|P(t, x)| \leq b(t)$ for almost all $t \in[0, T]$

We recover Caratheodory's existence theorem for ordinary differential equations [10]. Furthermore, the one-sided Lipschitz condition means that $P(t, \cdot)$ is Lipschitz continuous for all $t \in[0, T]$ (uniformly).

Remark 2 If $P$ is a monotone operator, i.e., for all $t \in[0, T]$, for all $x_{1}, x_{2} \in \mathbb{R}^{m}$,

$$
\left(y_{1}-y_{2}, x_{1}-x_{2}\right) \geq 0, \quad \forall y_{1} \in P\left(t, x_{1}\right), \forall y_{2} \in P\left(t, x_{2}\right),
$$

then $-P$ satisfies the one-sided Lipschitz condition.

3.2 The discrete setting

For simplicity, we suppose that $\Omega$ is a polyhedron. Let $\mathcal{T}$ be a simplicial mesh of $\Omega$ (triangles in 2D and tetrahedra in 3D). Let $\left\{x_{i}\right\}_{i \in \mathcal{N}}$ and $\left\{\phi_{i}\right\}_{i \in \mathcal{N}}$ be the nodes of the mesh and the associated scalar basis functions (continuous and piecewise affine), respectively. We denote by $\mathcal{N}^{D}$ the set of indices where a Dirichlet condition is enforced, and we set $\tilde{\mathcal{N}}:=\mathcal{N} \backslash \mathcal{N}^{D}$. The space of admissible displacements is approximated by the space

$$
V=\left\{v \in C^{0}(\bar{\Omega})^{d} ; v_{\mid T} \in\left(\mathbb{P}_{1}\right)^{d}, \forall T \in \mathcal{T}, \text { and } v\left(x_{i}\right)=0, \forall i \in \mathcal{N}^{D}\right\} .
$$

The space $V$ is spanned by $\left\{\phi_{i} e_{\alpha}\right\}_{i \in \tilde{\mathcal{N}}, 1 \leq \alpha \leq d}$, where $\left\{e_{\alpha}\right\}_{1 \leq \alpha \leq d}$ is the canonical basis of $\mathbb{R}^{d}$. Denote by $\mathcal{N}^{\mathrm{c}}$ the set of indices of contact nodes (that is, the nodes located on $\Gamma^{\mathrm{c}}$ which is fixed a priori) and by $\mathcal{N}^{\mathrm{i}}:=\tilde{\mathcal{N}} \backslash \mathcal{N}^{\mathrm{c}}$ the set of indices of the remaining nodes (see Fig. 2). Let $\left\{\nu_{i}\right\}_{i \in \mathcal{N}^{c}}$ and $\left\{\tau_{i, \alpha}\right\}_{i \in \mathcal{N}^{c}, 1 \leq \alpha \leq d-1}$ be the contact normal vectors and tangential vectors, respectively. We set

$$
\begin{gathered}
V^{\mathrm{i}}=\operatorname{span}\left(\left\{\phi_{i} e_{\alpha}\right\}_{i \in \mathcal{N}^{\mathrm{i}}, 1 \leq \alpha \leq d}\right), \\
V^{\mathrm{c}}=\operatorname{span}\left(\left\{\phi_{i} \nu_{i}\right\}_{i \in \mathcal{N}^{\mathrm{c}}}\right) \text { and } V^{\mathrm{f}}=\operatorname{span}\left(\left\{\phi_{i} \tau_{i, \alpha}\right\}_{i \in \mathcal{N}^{\mathrm{c}}, 1 \leq \alpha \leq d-1}\right) .
\end{gathered}
$$




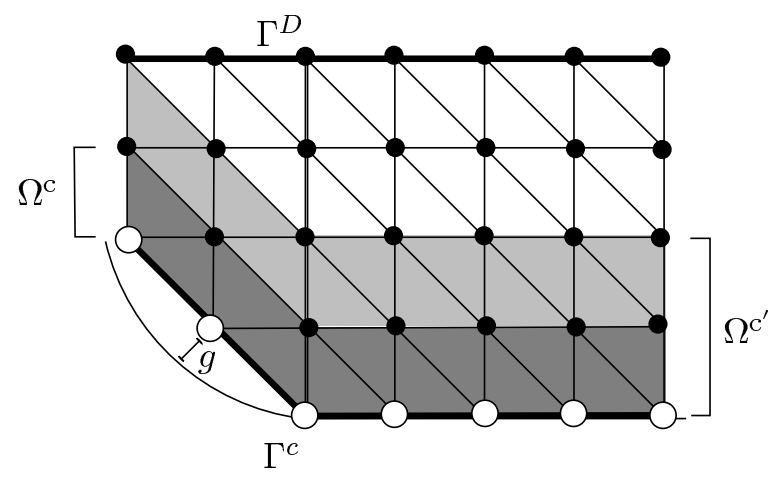

Fig. 2 Decomposition of the domain $\Omega$; bullets (resp., circles) indicate nodes indexed by elements of the set $\mathcal{N}^{\mathrm{i}}$ (resp., $\mathcal{N}^{\mathrm{c}}$ ). The open sets $\Omega^{c}$ and $\Omega^{c^{\prime}}$ are defined in Section 3.

Clearly, $V=V^{\mathrm{i}} \oplus V^{\mathrm{c}} \oplus V^{\mathrm{f}}$, so that any discrete function $v \in V$ can be decomposed as

$$
v=v_{\mathrm{i}}+v_{\mathrm{c}}+v_{\mathrm{f}} \quad \text { with } \quad v_{\mathrm{i}} \in V^{\mathrm{i}}, v_{\mathrm{c}} \in V^{\mathrm{c}}, v_{\mathrm{f}} \in V^{\mathrm{f}} .
$$

We also introduce the space $V^{*}:=V^{\mathrm{i}} \oplus V^{\mathrm{f}}$, so that any discrete function $v \in V$ can also be decomposed as

$$
v=v_{*}+v_{\mathrm{c}} \quad \text { with } \quad v_{*} \in V^{*}, v_{\mathrm{c}} \in V^{\mathrm{c}} .
$$

Let $(\cdot, \cdot)$ denote the $L^{2}$ inner product on $V$. Let $\|\cdot\|$ denote the norm associated with $(\cdot, \cdot)$. Herein, we always work in finite dimension on a fixed spatial mesh; the specific choice of the norm is therefore not critical. The present choice is made for simplicity. The standard mass term stems from the bilinear form

$$
m: L^{2}(\Omega)^{d} \times L^{2}(\Omega)^{d} \ni(v, w) \longmapsto \int_{\Omega} \rho v \cdot w \in \mathbb{R} .
$$

The key idea in the modified mass method is to remove the mass associated with the normal components at the contact nodes. We consider an approximate mass term associated with the bilinear form $m^{*}$ such that

$$
m^{*}\left(\phi_{i} \nu_{i}, w\right)=m^{*}\left(w, \phi_{i} \nu_{i}\right)=0, \quad \forall i \in \mathcal{N}^{\mathrm{c}}, \forall w \in V .
$$

Many choices are possible to build the rest of the mass term. In $[11,16]$, the authors devise various methods to preserve some features of the standard mass term (the total mass, the center of gravity, and the moments of inertia). Here, we focus for simplicity on the choice

$$
m^{*}: V \times V \ni(v, w) \longmapsto m\left(v_{*}, w_{*}\right) \in \mathbb{R} .
$$

We define the associated operator $M^{*}: V^{*} \rightarrow V^{*}$ such that,

$$
\left(M^{*} v_{*}, w_{*}\right)=m^{*}\left(v_{*}, w_{*}\right) \quad \forall\left(v_{*}, w_{*}\right) \in V^{*} \times V^{*} .
$$


We define the bilinear and linear forms

$$
\begin{aligned}
& a: H^{1}(\Omega)^{d} \times H^{1}(\Omega)^{d} \ni(v, w) \longmapsto \int_{\Omega} \epsilon(v): \mathcal{A}: \epsilon(w), \\
& l:[0, T] \times H^{1}(\Omega)^{d} \ni(t, v) \longmapsto \int_{\Omega} f(t) \cdot v+\int_{\Gamma^{N}} f_{N}(t) \cdot v .
\end{aligned}
$$

We define the linear operator $A: V \rightarrow V$ and the vector $L(t) \in V$ such that for all $v \in V$ and $w \in V$, and for all $t \in[0, T]$,

$$
(A v, w)=a(v, w), \quad(L(t), w)=l(t, w) .
$$

We also need $A^{\mathrm{c}}: V \rightarrow V^{\mathrm{c}}$, and $L^{\mathrm{c}}(t) \in V^{\mathrm{c}}$ such that for all $v \in V$ and all $w_{\mathrm{c}} \in V^{\mathrm{c}}$, and for all $t \in[0, T]$,

$$
\left(A^{\mathrm{c}} v, w_{\mathrm{c}}\right)=a\left(v, w_{\mathrm{c}}\right), \quad\left(L^{\mathrm{c}}(t), w_{\mathrm{c}}\right)=l\left(t, w_{\mathrm{c}}\right) .
$$

We define the constraint set

$$
K:=\left\{v \in V ; v\left(x_{i}\right) \cdot \nu_{i} \leq g\left(x_{i}\right), \forall i \in \mathcal{N}^{\mathrm{c}}\right\} .
$$

We define the unilateral contact term $I_{K}: V^{\mathrm{c}} \rightarrow \mathbb{R}$

$$
I_{K}\left(v_{\mathrm{c}}\right)= \begin{cases}0 & \text { if } v_{\mathrm{c}} \in K \\ +\infty & \text { if } v_{\mathrm{c}} \notin K\end{cases}
$$

The function $I_{K}$ is non-differentiable, but convex since $K$ is convex. Therefore, it is possible to define its subgradient $\partial I_{K}: V^{\mathrm{c}} \cap K \rightarrow \mathcal{P}^{*}\left(V^{\mathrm{c}}\right)$,

$$
\partial I_{K}\left(v_{\mathrm{c}}\right):=\left\{\gamma \in V^{\mathrm{c}} ; 0 \geq\left(\gamma, w_{\mathrm{c}}-v_{\mathrm{c}}\right) \forall w_{\mathrm{c}} \in V^{\mathrm{c}} \cap K\right\} .
$$

Now, we define the friction term $j: V \times V^{\mathrm{f}} \rightarrow \mathbb{R}$ such that

$$
j\left(v, w_{\mathrm{f}}\right)=\int_{\Gamma^{\mathrm{c}}} \mu\left|\sigma_{\nu}(v)\right|\left|w_{\mathrm{f}}\right|
$$

The function $j$ is non-differentiable with respect to its second argument, but convex, and its domain is $V^{\mathrm{f}}$. We can define its subgradient with respect to its second argument such that for all $z \in V, \partial_{2} j(z, \cdot): V^{\mathrm{f}} \rightarrow \mathcal{P}^{*}\left(V^{\mathrm{f}}\right)$ with

$$
\partial_{2} j\left(z, v_{\mathrm{f}}\right):=\left\{\gamma \in V^{\mathrm{f}} ; j\left(z, w_{\mathrm{f}}\right)-j\left(z, v_{\mathrm{f}}\right) \geq\left(\gamma, w_{\mathrm{f}}-v_{\mathrm{f}}\right) \forall w_{\mathrm{f}} \in V^{\mathrm{f}}\right\} .
$$

3.3 Formulation of the semi-discrete problem

We can now formulate the semi-discrete problem. Let $u_{*}^{0} \in V^{*}$ and $v_{*}^{0} \in V^{*}$ be suitable approximations of the initial displacement and velocity $u^{0}$ and $v^{0}$, respectively.

Problem 1 Seek $u \in C^{0}([0, T] ; K)$ such that $u_{*} \in C^{1}\left([0, T] ; V^{*}\right), \dot{u}_{*} \in A C\left([0, T] ; V^{*}\right)$, and the following differential inclusion holds true

$$
M^{*} \ddot{u}_{*} \in-A u-\partial_{2} j\left(u, \dot{u}_{\mathrm{f}}\right)-\partial I_{K}\left(u_{\mathrm{c}}\right)+L(t) \text { a.e. in }[0, T],
$$

with the initial conditions $u_{*}(0)=u_{*}^{0}$ and $\dot{u}_{*}(0)=v_{*}^{0}$ in $\Omega$. 
Remark 3 The velocity $\dot{u}_{*}$ is absolutely continuous. Therefore, it is differentiable almost everywhere, and the acceleration $\ddot{u}_{*}$ in (14) is well-defined. Moreover, $u_{\mathrm{c}} \in K$ so that $\partial I_{K}\left(u_{\mathrm{c}}\right)$ is well-defined.

To explicitate the link between the space semi-discrete Problem 1 and the continuous problem formulated in Section 2, we observe that (14) means that, for almost all $t \in[0, T]$, there exist $\lambda_{\mathrm{c}} \in \partial I_{K}\left(u_{\mathrm{c}}\right)$ and $\lambda_{\mathrm{f}} \in \partial_{2} j\left(u, \dot{u}_{\mathrm{f}}\right)$ such that

$$
M^{*} \ddot{u}_{*}+A u+\lambda_{\mathrm{c}}+\lambda_{\mathrm{f}}=L(t) .
$$

Therefore, the vectors $\lambda_{\mathrm{c}}$ and $\lambda_{\mathrm{f}}$ are discrete counterparts of the normal and tangential contact stresses. Furthermore, lumping the mass matrices, it is easy to verify that the definitions of $\partial I_{K}\left(u_{\mathrm{c}}\right)$ and $\partial_{2} j\left(u, \dot{u}_{\mathrm{f}}\right)$ imply that, for all $i \in \mathcal{N}^{\mathrm{c}}$,

$$
\begin{aligned}
& u_{\mathrm{c}}\left(x_{i}\right) \cdot \nu_{i} \leq g\left(x_{i}\right), \lambda_{\mathrm{c}}\left(x_{i}\right) \leq 0, \quad \lambda_{\mathrm{c}}\left(x_{i}\right)\left(u_{\mathrm{c}}\left(x_{i}\right) \cdot \nu_{i}-g\left(x_{i}\right)\right)=0, \\
& \left|\lambda_{\mathrm{f}}\left(x_{i}\right)\right| \leq \mu\left|\sigma_{\nu}(u)\left(x_{i}\right)\right| \\
& \lambda_{\mathrm{f}}\left(x_{i}\right)=-\mu \sigma_{\nu}(u)\left(x_{i}\right) \frac{\dot{u}_{\mathrm{f}}\left(x_{i}\right)}{\left|\dot{u}_{\mathrm{f}}\left(x_{i}\right)\right|} \quad \text { if } \dot{u}_{\mathrm{f}}\left(x_{i}\right) \neq 0 .
\end{aligned}
$$

Thus, we recover the discrete counterpart of the contact and friction conditions (3)-(5).

\subsection{Main results}

This section contains our main results concerning the space semi-discrete problem. We define the map $q:[0, T] \times V^{*} \rightarrow V^{\mathrm{c}} \cap K$ such that for all $t \in[0, T]$ and for all $v_{*} \in V^{*}$, $v_{\mathrm{c}}=q\left(t, v_{*}\right) \in V^{\mathrm{c}} \cap K$ solves the following variational inequality

$$
a\left(v_{\mathrm{c}}, w_{\mathrm{c}}-v_{\mathrm{c}}\right) \geq l\left(t, w_{\mathrm{c}}-v_{\mathrm{c}}\right)-a\left(v_{*}, w_{\mathrm{c}}-v_{\mathrm{c}}\right) \quad \forall w_{\mathrm{c}} \in V^{\mathrm{C}} \cap K, \forall t \in[0, T] .
$$

This variational inequality is well-posed since it is equivalent to the minimization of a strictly convex functional over a convex set. We first examine the properties of the $\operatorname{map} q$.

Lemma 1 For all $v_{*} \in V^{*}$, the map $t \mapsto q\left(t, v_{*}\right)$ is Lipschitz continuous, and its Lipschitz constant is uniformly bounded in $v_{*}$. For all $t \in[0, T]$, the map $v_{*} \mapsto q\left(t, v_{*}\right)$ is Lipschitz continuous, and its Lipschitz constant is uniformly bounded in $t$.

Proof Let $t_{1}, t_{2} \in[0, T]$ and $v_{*}, w_{*} \in V^{*}$. Set $v_{\mathrm{c}}=q\left(t_{1}, v_{*}\right)$ and $w_{\mathrm{c}}=q\left(t_{2}, w_{*}\right)$. Owing to $(18)$,

$$
a\left(v_{\mathrm{c}}-w_{\mathrm{c}}, v_{\mathrm{c}}-w_{\mathrm{c}}\right) \leq a\left(v_{*}-w_{*}, w_{\mathrm{c}}-v_{\mathrm{c}}\right)+l\left(t_{1}-t_{2}, v_{\mathrm{c}}-w_{\mathrm{c}}\right) .
$$

Since

$l\left(t_{1}-t_{2}, v_{\mathrm{c}}-w_{\mathrm{c}}\right)=\int_{\Omega}\left(f\left(t_{1}\right)-f\left(t_{2}\right)\right) \cdot\left(v_{\mathrm{c}}-w_{\mathrm{c}}\right)+\int_{\Gamma^{N}}\left(f_{N}\left(t_{1}\right)-f_{N}\left(t_{2}\right)\right) \cdot\left(v_{\mathrm{c}}-w_{\mathrm{c}}\right)$,

and since $f \in W^{1, \infty}\left(0, T ; L^{2}(\Omega)^{d}\right)$ and $f_{N} \in W^{1, \infty}\left(0, T ; L^{2}\left(\Gamma^{N}\right)^{d}\right)$, there exists a constant $c_{l}$ such that

$$
l\left(t_{1}-t_{2}, v_{\mathrm{c}}-w_{\mathrm{c}}\right) \leq c_{l}\left|t_{1}-t_{2}\right||| v_{\mathrm{c}}-w_{\mathrm{c}} \| .
$$


Moreover, the bilinear form $a$ being continuous (with constant $c_{a}$ ) and elliptic (with constant $\alpha$ ) for the norm $\|\cdot\|$, a straightforward calculation yields

$$
\alpha\left\|v_{\mathrm{c}}-w_{\mathrm{c}}\right\| \leq c_{a}\left\|v_{*}-w_{*}\right\|+c_{l}\left|t_{1}-t_{2}\right|,
$$

which proves the desired regularity for $q$.

We now reformulate the differential inclusion (14) using the map $q$.

Lemma 2 The differential inclusion (14) is equivalent to

$$
\begin{array}{ll}
M^{*} \ddot{u}_{*} \in-A^{*}\left(u_{*}+q\left(t, u_{*}\right)\right)-\partial_{2} j\left(u_{*}+q\left(t, u_{*}\right), \dot{u}_{\mathrm{f}}\right)+L^{*}(t), & \text { a.e.in }[0, T], \\
u_{\mathrm{c}}=q\left(t, u_{*}\right), & \forall t \in[0, T],
\end{array}
$$

Proof Distinguishing components in $V^{*}$ and $V^{\mathrm{c}}$, the inclusion (14) is equivalently split into the following inclusions

$$
\begin{array}{ll}
M^{*} \ddot{u}_{*} \in-A^{*} u-\partial_{2} j\left(u, \dot{u}_{\mathrm{f}}\right)+L^{*}(t) & \text { a.e. in }[0, T], \\
0 \in-A^{\mathrm{c}} u-\partial I_{K}\left(u_{\mathrm{c}}\right)+L^{\mathrm{c}}(t) & \text { a.e. in }[0, T] .
\end{array}
$$

Consider (23). By continuity, the inclusion (23) is valid for all $t \in[0, T]$. For convenience, we recast it as a variational inequality,

$$
a\left(u, v_{\mathrm{c}}-u_{\mathrm{c}}\right) \geq l\left(t, v_{\mathrm{c}}-u_{\mathrm{c}}\right) \quad \forall t \in[0, T], \forall v_{\mathrm{c}} \in V^{\mathrm{c}} \cap K,
$$

or, equivalently,

$$
a\left(u_{\mathrm{c}}, v_{\mathrm{c}}-u_{\mathrm{c}}\right) \geq l\left(t, v_{\mathrm{c}}-u_{\mathrm{c}}\right)-a\left(u_{*}, v_{\mathrm{c}}-u_{\mathrm{c}}\right) \quad \forall t \in[0, T], \forall v_{\mathrm{c}} \in V^{\mathrm{c}} \cap K .
$$

Hence $u_{\mathrm{c}}=q\left(t, u_{*}\right)$ so that the system (22)-(23) is equivalent to the system (20)-(21).

We can now state our main existence result for Problem 1.

Theorem 2 There exists a unique solution u to Problem 1. Furthermore, $u_{\mathrm{c}} \in W^{1, \infty}\left(0, T ; V^{\mathrm{c}}\right)$.

Proof (i) To prove the existence of a solution, we rewrite the second-order differential inclusion (14) as a first-order differential inclusion. We define the single-valued map $S:[0, T] \times V^{*} \times V^{*} \rightarrow V^{*} \times V^{*}$ such that, for all $t \in[0, T]$, for all $v_{*}, w_{*} \in V^{*}$,

$$
S\left(t, v_{*}, w_{*}\right)=\left(\begin{array}{c}
w_{*} \\
-A^{*}\left(v_{*}+q\left(t, v_{*}\right)\right)+L^{*}(t)
\end{array}\right),
$$

and the set-valued map $P:[0, T] \times V^{*} \times V^{*} \rightarrow\{0\} \times \mathcal{P}^{*}\left(V^{*}\right)$ such that

$$
P\left(t, v_{*}, w_{*}\right)=\left(\begin{array}{c}
0 \\
-\partial_{2} j\left(v_{*}+q\left(t, v_{*}\right), w_{\mathrm{f}}\right)
\end{array}\right) .
$$

We define also the linear single-valued map $D: V^{*} \times V^{*} \rightarrow V^{*} \times V^{*}$ such that

$$
D\left(v_{*}, w_{*}\right)=\left(\begin{array}{c}
v_{*} \\
M^{*} w_{*}
\end{array}\right) .
$$

Setting $X(t)=\left(\begin{array}{c}u_{*}(t) \\ \dot{u}_{*}(t)\end{array}\right) \in V^{*} \times V^{*}$, the differential inclusion (20) can be recast as

$$
D \dot{X}(t) \in S(t, X(t))+P(t, X(t)) \text {. }
$$


We equip the product space $V^{*} \times V^{*}$ with the product norm.

(ii) The operator $S$ is a single-valued map. Since $q(t, \cdot)$ is continuous and $q(\cdot, x)$ is Lipschitz continuous, the operator $S$ satisfies the hypotheses of Theorem 1 (see Remark 1).

(iii) We now examine the operator $P$. This operator is a closed convex set-valued map (owing to the properties of the subgradients of convex functions). Since $q(t, \cdot)$ is continuous and $\partial_{2} j(\cdot, \cdot)$ is upper semi-continuous (see the example given by (3.1)), the map $P(t, \cdot)$ is upper semi-continuous. Hence, Hypothesis 1 of Theorem 1 holds true. Since $q(\cdot, x)$ is Lipschitz continuous, Hypotheses 2 and 3 of this theorem are also satisfied. Next, we check the one-sided Lipschitz condition (10). Let $\left(u_{*}^{1}, u_{*}^{2}, v_{*}^{1}, v_{*}^{2}\right) \in$ $\left(V^{*}\right)^{4}$ and let $t \in[0, T]$. Set $u^{1}=u_{*}^{1}+q\left(t, u_{*}^{1}\right)$ and $u^{2}=u_{*}^{2}+q\left(t, u_{*}^{2}\right)$. Let $\gamma_{1} \in$ $-\partial_{2} j\left(u^{1}, v_{\mathrm{f}}^{1}\right)$ and let $\gamma_{2} \in-\partial_{2} j\left(u^{2}, v_{\mathrm{f}}^{2}\right)$. Using the definition of the subgradient, a reverse triangle inequality, norm equivalence in finite dimension, and the fact that $q(t, \cdot)$ is Lipschitz, we infer

$$
\begin{aligned}
\left(\gamma_{2}-\gamma_{1}, v_{*}^{1}-v_{*}^{2}\right) & \leq j\left(u^{1}, v_{\mathrm{f}}^{1}\right)-j\left(u^{1}, v_{\mathrm{f}}^{2}\right)+j\left(u^{2}, v_{\mathrm{f}}^{2}\right)-j\left(u^{2}, v_{\mathrm{f}}^{1}\right) \\
& \leq \int_{\Gamma^{\mathrm{c}}} \mu\left(\left|\sigma_{\nu}\left(u^{1}\right)\right|-\left|\sigma_{\nu}\left(u^{2}\right)\right|\right)\left(\left|v_{*}^{1}\right|-\left|v_{*}^{2}\right|\right) \\
& \leq \int_{\Gamma^{\mathrm{c}}} \mu|| \sigma_{\nu}\left(u^{1}\right)|-| \sigma_{\nu}\left(u^{2}\right)|||| v_{*}^{1}|-| v_{*}^{2}|| \\
& \leq \int_{\Gamma^{\mathrm{c}}} \mu\left|\sigma_{\nu}\left(u^{1}\right)-\sigma_{\nu}\left(u^{2}\right) \| v_{*}^{1}-v_{*}^{2}\right| \\
& \lesssim\left\|u^{1}-u^{2}\right\|\left\|v_{*}^{1}-v_{*}^{2}\right\| \\
& \lesssim\left(\left\|u_{*}^{1}-u_{*}^{2}\right\|+\left\|q\left(t, u_{*}^{1}\right)-q\left(t, u_{*}^{2}\right)\right\|\right)\left\|v_{*}^{1}-v_{*}^{2}\right\| \\
& \lesssim\left\|u_{*}^{1}-u_{*}^{2}\right\|\left\|v_{*}^{1}-v_{*}^{2}\right\| \lesssim\left\|u_{*}^{1}-u_{*}^{2}\right\|^{2}+\left\|v_{*}^{1}-v_{*}^{2}\right\|^{2} .
\end{aligned}
$$

Therefore, $P$ satisfies the one-sided Lipschitz condition.

(iv) Owing to Theorem 1 , there exists a unique $X \in A C\left([0, T] ; V^{*} \times V^{*}\right)$ satisfying (26) with the initial condition $X(0)=\left(\begin{array}{c}u_{*}^{0} \\ v_{*}^{0}\end{array}\right)$. Therefore, there exists a unique $u_{*} \in$ $C^{1}\left(0, T ; V^{*}\right)$ such that $\dot{u}_{*} \in A C\left([0, T] ; V^{*}\right)$ satisfying $(20)$ with the initial conditions $u_{*}(0)=u_{*}^{0}$ and $\dot{u}_{*}(0)=v_{*}^{0}$. Owing to $(21), u=u_{*}+u_{\mathrm{c}}=u_{*}+q\left(t, u_{*}\right)$. Therefore, Problem 1 has a unique solution and it is clear that $u_{\mathrm{c}}=q\left(t, u_{*}\right) \in W^{1, \infty}\left(0, T ; V^{\mathrm{c}}\right)$.

We conclude this section with the energy balance.

Theorem 3 For all $t_{0} \in[0, T]$, the following energy balance holds true:

$$
E\left(u\left(t_{0}\right)\right)-E(u(0))=\int_{0}^{t_{0}}\left\{l(t, \dot{u}(t))-j\left(u(t), \dot{u}_{\mathrm{f}}(t)\right)\right\} d t
$$

where $E(v)=\frac{1}{2}\left(m\left(\dot{v}_{*}, \dot{v}_{*}\right)+a(v, v)\right)$.

Proof We recast the differential inclusion (20) as a variational inequality,

$$
\begin{aligned}
m\left(\ddot{u}_{*}, v_{*}-\dot{u}_{*}\right)+a\left(u, v_{*}-\dot{u}_{*}\right)+j\left(u, v_{\mathrm{f}}\right)-j\left(u, \dot{u}_{\mathrm{f}}\right) & \\
& \geq l\left(t, v_{*}-\dot{u}_{*}\right) \quad \forall v_{*} \in V^{*}, \text { a.e. in }[0, T] .
\end{aligned}
$$


Taking $v_{*}=0$ and then $v_{*}=2 \dot{u}_{*}$ in the above inequality, we obtain

$$
m\left(\ddot{u}_{*}, \dot{u}_{*}\right)+a\left(u, \dot{u}_{*}\right)+j\left(u, \dot{u}_{\mathrm{f}}\right)=l\left(t, \dot{u}_{*}\right) \quad \text { a.e. on }[0, T] .
$$

Recalling that the family $\left\{\phi_{i} \nu_{i}\right\}_{i \in \mathcal{N}^{c}}$ is a basis of $V^{\mathrm{c}}$, we decompose $u_{\mathrm{c}}$ on this basis yielding $u_{\mathrm{c}}=\sum_{i \in \mathcal{N}^{\mathrm{c}}} u_{i} \phi_{i} \nu_{i}$. Define $C_{i}^{0}:=\left\{t \in[0, T] ; u_{i}=0\right\}$ and $C_{i}^{-}:=\{t \in$ $\left.[0, T] ; u_{i}<0\right\}$. The sets $C_{i}^{0}$ and $C_{i}^{-}$are respectively closed and open, and they form a partition of $[0, T]$. On $\operatorname{int}\left(C_{i}^{0}\right), \dot{u}_{i} \phi_{i} \nu_{i}=0$. Owing to (23), $a\left(u, \dot{u}_{i} \phi_{i} \nu_{i}\right)=0$ on $C_{i}^{-}$. Finally, $a\left(u, \dot{u}_{i} \phi_{i} \nu_{i}\right)=0$ on $\operatorname{int}\left(C_{i}^{0}\right) \cup C_{i}^{-}$, and hence almost everywhere (since an open set in $\mathbb{R}$ is a countable union of open intervals, so that its boundary has zero measure). Hence,

$$
a\left(u, \dot{u}_{\mathrm{c}}\right)=l\left(t, \dot{u}_{\mathrm{c}}\right) \quad \text { a.e. on }[0, T] .
$$

Using (30), we obtain

$$
m\left(\ddot{u}_{*}, \dot{u}_{*}\right)+a(u, \dot{u})+j\left(u, \dot{u}_{\mathrm{f}}\right)=l(t, \dot{u}) \quad \text { a.e. on }[0, T] .
$$

Since $\dot{u}$ is absolutely continuous in time, by integrating in time (31), we obtain (27).

\section{Fully discrete formulation}

In this section, we discretize the space semi-discrete problem with an implicit time scheme. We discretize the elastodynamic part with an implicit Newmark scheme (trapezoidal rule), while the unilateral contact and friction conditions are enforced in an implicit way. This choice of time discretization is very common. It is for instance employed in [11]. At each time step, we have thus to solve a nonlinear problem similar to a static friction problem. It is well-known that such a problem may have several solutions. Here we prove that, under a certain condition on the discretization parameters of CFL-type, the fully discrete problem is well-posed. We also derive the energy balance of this time-integration scheme.

For simplicity, the interval $[0, T]$ is divided into $N$ equal subintervals of length $\Delta t$. We set $t^{n}=n \Delta t$ and denote by $u^{n}, v^{n}$, and $a^{n}$ the approximations of $u\left(t^{n}\right), \dot{u}\left(t^{n}\right)$, and $\ddot{u}\left(t^{n}\right)$, respectively. We define the convex combination $\square^{n+\alpha}:=(1-\alpha) \square^{n}+\alpha \square^{n+1}$, where $\square$ stands for $u, v$, a or $t$, and $\alpha \in[0,1]$. In this section, the notation $A \lesssim B$ means that $A \leq c B$ with a constant $c$ independent of $h$ and $\Delta t$.

Let $\mathcal{T}^{\mathrm{c}} \subset \mathcal{T}$ be the set of simplices such that at least one vertex is a contact node. We set $\Omega^{\mathrm{c}}=\operatorname{int}\left(\cup_{T \in \mathcal{T}} \bar{T}\right)$. Let $\mathcal{T}^{c^{\prime}} \subset \mathcal{T}$ be the set of simplices such that at least one vertex belongs to $\overline{\Omega^{\mathrm{c}}}$. We set $\Omega^{c^{\prime}}=\operatorname{int}\left(\cup_{T \in \mathcal{T}}{ }^{\prime} \bar{T}\right)$ (see Fig. 2). We define

$$
h_{\mathrm{c}}=\min _{T \in \mathcal{T}^{\mathrm{c}}} \operatorname{diam}(T) \text { and } h_{c^{\prime}}=\min _{T \in \mathcal{T}^{c^{\prime}}} \operatorname{diam}(T),
$$

where $\operatorname{diam}(T)$ denotes the diameter of the simplex $T$. Observe that $h_{\mathrm{c}}$ and $h_{\mathrm{c}^{\prime}}$ are defined using a minimum.

Let us recall some classical discrete trace and inverse inequalities (see, e.g., [23] and $[9])$. For all $v_{\mathrm{c}} \in V^{\mathrm{c}}$,

$$
\left\|v_{\mathrm{c}}\right\|_{L^{2}\left(\Gamma^{\mathrm{c}}\right)^{d}} \leq \frac{1}{\sqrt{h_{\mathrm{c}}}}\left\|v_{\mathrm{c}}\right\|_{L^{2}\left(\Omega^{\mathrm{c}}\right)^{d}}
$$




$$
\left|v_{\mathrm{c}}\right|_{H^{1}\left(\Omega^{\mathrm{c}}\right)^{d}}=\left\|\nabla v_{\mathrm{c}}\right\|_{L^{2}\left(\Omega^{\mathrm{c}}\right)^{d \times d}} \leq \frac{1}{h_{\mathrm{c}}}\left\|v_{\mathrm{c}}\right\|_{L^{2}\left(\Omega^{\mathrm{c}}\right)^{d}} .
$$

The same inequalities hold when $\Omega^{\mathrm{c}}$ is replaced by $\Omega^{\mathrm{c}^{\prime}}$, and $h_{\mathrm{c}}$ by $h_{\mathrm{c}^{\prime}}$. We define the operator $q^{n}: V^{*} \rightarrow V^{\mathrm{c}}$, such that for all $0 \leq n \leq N$,

$$
q^{n}\left(v_{*}\right)=q\left(t^{n}, v_{*}\right) \quad \forall v_{*} \in V^{*},
$$

where the map $q$ is defined in Section 3.4.

Lemma 3 The function $q^{n}: V^{*} \rightarrow V^{\mathrm{c}}$ is Lipschitz continuous. More precisely,

$$
\left|q^{n}\left(v_{*}\right)-q^{n}\left(w_{*}\right)\right|_{H^{1}\left(\Omega^{c}\right)^{d}} \lesssim\left|v_{*}-w_{*}\right|_{H^{1}\left(\Omega^{c^{\prime}}\right)^{d}} \quad \forall v_{*}, w_{*} \in V^{*} .
$$

Proof Let $v_{*}, w_{*} \in V^{*}$. Set $v_{\mathrm{c}}=q^{n}\left(v_{*}\right)$ and $w_{\mathrm{c}}=q^{n}\left(w_{*}\right)$. Owing to (19),

$$
a\left(v_{\mathrm{c}}-w_{\mathrm{c}}, v_{\mathrm{c}}-w_{\mathrm{c}}\right) \leq a\left(v_{*}-w_{*}, w_{\mathrm{c}}-v_{\mathrm{c}}\right) .
$$

Since $v_{\mathrm{c}}$ and $w_{\mathrm{c}}$ are zero outside $\Omega^{\mathrm{c}}, a\left(v_{\mathrm{c}}-w_{\mathrm{c}}, v_{\mathrm{c}}-w_{\mathrm{c}}\right) \gtrsim\left|v_{\mathrm{c}}-w_{\mathrm{c}}\right|_{H^{1}\left(\Omega^{\mathrm{c}}\right)^{d}}^{2}$, and $a\left(v_{*}-w_{*}, w_{\mathrm{c}}-v_{\mathrm{C}}\right)=a\left(\left(v_{*}-w_{*}\right) 1_{\Omega^{c^{\prime}}}, w_{\mathrm{C}}-v_{\mathrm{c}}\right) \lesssim\left|v_{*}-w_{*}\right|_{H^{1}\left(\Omega^{c^{\prime}}\right)^{d}}\left|v_{\mathrm{c}}-w_{\mathrm{c}}\right|_{H^{1}\left(\Omega^{\mathrm{c}}\right)^{d}}$, whence the assertion.

We can now formulate the fully discrete problem.

Problem 2 Seek $u^{n+1} \in V, v_{*}^{n+1} \in V^{*}$, and $a_{*}^{n+1} \in V^{*}$ such that

$$
\begin{aligned}
& M^{*} a_{*}^{n+1} \in-A^{*} u^{n+1}-\partial_{2} j\left(u^{n+1}, v_{\mathrm{f}}^{n+1}\right)+L^{*}\left(t^{n+1}\right), \\
& u_{\mathrm{c}}^{n+1}=q^{n+1}\left(u_{*}^{n+1}\right), \\
& u_{*}^{n+1}=u_{*}^{n}+\Delta t v_{*}^{n}+\frac{\Delta t^{2}}{2} a_{*}^{n+\frac{1}{2}}, \\
& v_{*}^{n+1}=v_{*}^{n}+\Delta t \mathrm{a}_{*}^{n+\frac{1}{2}} .
\end{aligned}
$$

To begin with, we reformulate Problem 2 by eliminating $v_{*}^{n+1}$ and $a_{*}^{n+1}$. We set $\delta_{*}^{n}:=-u_{*}^{n}-\frac{\Delta t}{2} v_{*}^{n}$ and $\varepsilon_{*}^{n}:=-u_{*}^{n}-\Delta t v_{*}^{n}-\frac{\Delta t^{2}}{4} a_{*}^{n}$, and we rewrite $v_{*}^{n+1}$ and $a_{*}^{n+1}$ as

$$
\begin{aligned}
& v_{*}^{n+1}=\frac{2}{\Delta t}\left(u_{*}^{n+1}+\delta_{*}^{n}\right), \\
& a_{*}^{n+1}=\frac{4}{\Delta t^{2}}\left(u_{*}^{n+1}+\varepsilon_{*}^{n}\right) .
\end{aligned}
$$

Next, we define the linear operator $\tilde{A}^{*}: V^{*} \rightarrow V^{*}$ and the vector $\tilde{L}^{n+1} \in V^{*}$ such that, $\forall v_{*} \in V^{*}$,

$$
\begin{aligned}
& \tilde{A}^{*} v_{*}:=A^{*} v_{*}+\frac{1}{4 \Delta t^{2}} M^{*} v_{*}, \\
& \left(\tilde{L}^{n+1}, v_{*}\right):=L^{*}\left(t^{n+1}\right)-\frac{1}{4 \Delta t^{2}} M^{*} \varepsilon_{*}^{n+1} .
\end{aligned}
$$

Then, using (37), it is straightforward to turn (36) into

$$
0 \in \tilde{A}^{*} u_{*}^{n+1}+\partial_{2} j\left(u_{*}^{n+1}+q^{n+1}\left(u_{*}^{n+1}\right), \frac{2}{\Delta t}\left(u_{*}^{n+1}+\delta_{*}^{n}\right)\right)-\tilde{L}^{n+1}+A^{*} q^{n+1}\left(u_{*}^{n+1}\right) \text {. }
$$

Observe that the last term on the right-hand side of (40) involves the operator $A^{*}$ (and not $\left.\tilde{A}^{*}\right)$ owing to $(11)$ and the fact that $q^{n+1}\left(u_{*}^{n+1}\right) \in V^{\mathrm{c}}$. 
Theorem 4 Problem 2 has a unique solution under the CFL-condition

$$
\frac{\Delta t}{h_{c^{\prime}}} \lesssim 1
$$

Proof Define the map $\Phi^{n}: V^{*} \rightarrow V^{*}$ such that for all $\hat{v}_{*} \in V^{*}, v_{*}=\Phi^{n}\left(\hat{v}_{*}\right)$ satisfies

$$
0 \in \tilde{A}^{*} v_{*}+\partial_{2} j\left(\hat{v}, \frac{2}{\Delta t}\left(v_{*}+\delta_{*}^{n}\right)\right)-\tilde{L}^{n+1}+A^{*} \hat{v}_{\mathrm{c}}
$$

where $\hat{v}_{\mathrm{c}}:=q^{n+1}\left(\hat{v}_{*}\right)$ and $\hat{v}:=\hat{v}_{*}+\hat{v}_{\mathrm{c}}$, so that (40) amounts to seeking a fixed-point for $\Phi^{n}$. Setting $y_{*}:=\frac{2}{\Delta t}\left(v_{*}+\delta_{*}^{n}\right)$, we rewrite the above inclusion as a variational inequality,

$$
\tilde{a}\left(v_{*}, z_{*}-y_{*}\right)+j\left(\hat{v}, z_{\mathrm{f}}\right)-j\left(\hat{v}, \dot{v}_{\mathrm{f}}\right) \geq l^{n+1}\left(z_{*}-y_{*}\right)-a\left(\hat{v}_{\mathrm{c}}, z_{*}-y_{*}\right), \forall z_{*} \in V^{*},
$$

where we have set $\tilde{a}\left(v_{*}, w_{*}\right):=\left(\tilde{A}^{*} v_{*}, w_{*}\right)$ and $l^{n+1}\left(v_{*}\right):=\left(L\left(t^{n+1}\right), v_{*}\right)$. Taking $z_{*}:=\frac{2}{\Delta t}\left(w_{*}+\delta_{*}^{n}\right)$ in $(43)$, then dividing by $\frac{2}{\Delta t}$, we obtain for all $w_{*} \in V^{*}$,

$$
\tilde{a}\left(v_{*}, w_{*}-v_{*}\right)+j\left(\hat{v}, w_{\mathrm{f}}+\delta_{\mathrm{f}}^{n}\right)-j\left(\hat{v}, v_{\mathrm{f}}+\delta_{\mathrm{f}}^{n}\right) \geq l^{n+1}\left(w_{*}-v_{*}\right)-a\left(\hat{v}_{\mathrm{c}}, w_{*}-v_{*}\right) .
$$

The variational inequality (44) has one and only one solution. Indeed, it is equivalent to the minimization of a strictly convex functional. The map $\Phi^{n}$ is thus well-defined. Now we shall prove that $\Phi^{n}$ is a contraction under the CFL condition (41). Let $\hat{v}_{*} \in V^{*}$ and $\hat{w}_{*} \in V^{*}$. Set $v_{*}:=\Phi^{n}\left(\hat{v}_{*}\right)$ and $w_{*}:=\Phi^{n}\left(\hat{w}_{*}\right)$. Using (44), a straightforward calculation yields

$$
\begin{aligned}
\tilde{a}\left(v_{*}-w_{*}, v_{*}-w_{*}\right) \leq & j\left(\hat{v}, w_{\mathrm{f}}+\delta_{\mathrm{f}}^{n}\right)-j\left(\hat{w}, w_{\mathrm{f}}+\delta_{\mathrm{f}}^{n}\right) \\
& -j\left(\hat{v}, v_{\mathrm{f}}+\delta_{\mathrm{f}}^{n}\right)+j\left(\hat{w}, v_{\mathrm{f}}+\delta_{\mathrm{f}}^{n}\right)-a\left(\hat{v}_{\mathrm{c}}-\hat{w}_{\mathrm{c}}, v_{*}-w_{*}\right) .
\end{aligned}
$$

Using the ellipticity of $m$ and $a$,

$$
\tilde{a}\left(v_{*}-w_{*}, v_{*}-w_{*}\right) \gtrsim \frac{4}{\Delta t^{2}}\left\|v_{*}-w_{*}\right\|_{L^{2}(\Omega)^{d}}^{2}+\left|v_{*}-w_{*}\right|_{H^{1}(\Omega)^{d}}^{2} .
$$

Using a reverse triangle inequality,

$$
\begin{aligned}
j\left(\hat{v}, w_{\mathrm{f}}+\delta_{\mathrm{f}}^{n}\right) & -j\left(\hat{w}, w_{\mathrm{f}}+\delta_{\mathrm{f}}^{n}\right)-j\left(\hat{v}, v_{\mathrm{f}}+\delta_{\mathrm{f}}^{n}\right)+j\left(\hat{w}, v_{\mathrm{f}}+\delta_{\mathrm{f}}^{n}\right) \\
& \leq \int_{\Gamma^{\mathrm{c}}} \mu\left|\sigma_{\nu}(\hat{v})-\sigma_{\nu}(\hat{w})\right||| w_{\mathrm{f}}+\delta_{\mathrm{f}}^{n}|-| v_{\mathrm{f}}+\delta_{\mathrm{f}}^{n}|| \\
& \leq \int_{\Gamma^{\mathrm{c}}}|\mu|\left|\sigma_{\nu}(\hat{v})-\sigma_{\nu}(\hat{w})\right|\left|v_{\mathrm{f}}-w_{\mathrm{f}}\right| \\
& \lesssim \int_{\Gamma^{\mathrm{c}}}\left|\sigma_{\nu}(\hat{v})-\sigma_{\nu}(\hat{w})\right|\left|v_{\mathrm{f}}-w_{\mathrm{f}}\right| .
\end{aligned}
$$

Using the Cauchy-Schwarz inequality and the trace inequality (32),

$$
\begin{aligned}
j\left(\hat{v}, w_{\mathrm{f}}+\delta_{\mathrm{f}}^{n}\right)- & j\left(\hat{w}, w_{\mathrm{f}}+\delta_{\mathrm{f}}^{n}\right)-j\left(\hat{v}, v_{\mathrm{f}}+\delta_{\mathrm{f}}^{n}\right)+j\left(\hat{w}, v_{\mathrm{f}}+\delta_{\mathrm{f}}^{n}\right) \\
& \lesssim\left\|\sigma_{\nu}(\hat{v})-\sigma_{\nu}(\hat{w})\right\|_{L^{2}\left(\Gamma^{c}\right)}\left\|v_{\mathrm{f}}-w_{\mathrm{f}}\right\|_{L^{2}\left(\Gamma^{\mathrm{c}}\right)^{d}} \\
& \lesssim \frac{1}{h_{\mathrm{c}}}\left\|\sigma_{\nu}(\hat{v})-\sigma_{\nu}(\hat{w})\right\|_{L^{2}\left(\Omega^{\mathrm{c}}\right)}\left\|v_{*}-w_{*}\right\|_{L^{2}\left(\Omega^{\mathrm{c}}\right)^{d}} \\
& \lesssim \frac{1}{h_{\mathrm{c}}}|\hat{v}-\hat{w}|_{H^{1}\left(\Omega^{\mathrm{c}}\right)^{d}}\left\|v_{*}-w_{*}\right\|_{L^{2}\left(\Omega^{\mathrm{c}}\right)^{d}} .
\end{aligned}
$$


Furthermore, using (35) and the inverse inequality (33),

$$
\begin{aligned}
|\hat{v}-\hat{w}|_{H^{1}\left(\Omega^{\mathrm{c}}\right)^{d}} & =\left|\hat{v}_{\mathrm{c}}-\hat{w}_{\mathrm{c}}\right|_{H^{1}\left(\Omega^{\mathrm{c}}\right)^{d}}+\left|\hat{v}_{*}-\hat{w}_{*}\right|_{H^{1}\left(\Omega^{\mathrm{c}}\right)^{d}} \\
& =\left|q^{n+1}\left(\hat{v}_{*}\right)-q^{n+1}\left(\hat{w}_{*}\right)\right|_{H^{1}\left(\Omega^{\mathrm{c}}\right)^{d}}+\left|\hat{v}_{*}-\hat{w}_{*}\right|_{H^{1}\left(\Omega^{\mathrm{c}}\right)^{d}} \\
& \lesssim\left|\hat{v}_{*}-\hat{w}_{*}\right|_{H^{1}\left(\Omega^{c^{\prime}}\right)^{d}}+\left|\hat{v}_{*}-\hat{w}_{*}\right|_{H^{1}\left(\Omega^{\mathrm{c}}\right)^{d}} \\
& \lesssim\left|\hat{v}_{*}-\hat{w}_{*}\right|_{H^{1}\left(\Omega^{c^{\prime}}\right)^{d}} \lesssim \frac{1}{h_{c^{\prime}}}\left\|v_{*}-w_{*}\right\|_{L^{2}\left(\Omega^{c^{\prime}}\right)^{d}} .
\end{aligned}
$$

Collecting inequalities (47) and (48), and since $h_{c^{\prime}} \leq h_{\mathrm{c}}$,

$$
\begin{aligned}
j\left(\hat{v}, w_{\mathrm{f}}+\delta_{\mathrm{f}}^{n}\right)-j\left(\hat{w}, w_{\mathrm{f}}+\delta_{\mathrm{f}}^{n}\right)-j\left(\hat{v}, v_{\mathrm{f}}+\delta_{\mathrm{f}}^{n}\right)+j\left(\hat{w}, v_{\mathrm{f}}+\delta_{\mathrm{f}}^{n}\right) \\
\leq \frac{1}{h_{c^{\prime}}^{2}}\left\|\hat{v}_{*}-\hat{w}_{*}\right\|_{L^{2}(\Omega)^{d}}\left\|v_{*}-w_{*}\right\|_{L^{2}\left(\Omega^{\mathrm{c}}\right)^{d}} .
\end{aligned}
$$

Using the inverse inequality (33),

$$
a\left(\hat{v}_{\mathrm{c}}-\hat{w}_{\mathrm{c}}, w_{*}-v_{*}\right) \lesssim \frac{1}{h_{c^{\prime}}^{2}}\left\|\hat{w}_{\mathrm{C}}-\hat{v}_{\mathrm{c}}\right\|_{L^{2}(\Omega)^{d}}\left\|v_{*}-w_{*}\right\|_{L^{2}(\Omega)^{d}}
$$

Collecting these different estimates,

$$
\left\|\Phi^{n}\left(\hat{v}_{*}\right)-\Phi^{n}\left(\hat{w}_{*}\right)\right\|_{L^{2}(\Omega)^{d}}=\left\|v_{*}-w_{*}\right\|_{L^{2}(\Omega)^{d}} \lesssim\left(\frac{\Delta t}{h_{c^{\prime}}}\right)^{2}\left\|\hat{v}_{*}-\hat{w}_{*}\right\|_{L^{2}(\Omega)^{d}} .
$$

Hence, if the ratio $\frac{\Delta t}{h_{c^{\prime}}}$ is sufficiently small, the mapping $\Phi^{n}$ is a contraction. The Banach fixed-point theorem guarantees that the problem has a unique solution.

Remark 4 In the above proof, the inertial term is essential. By strengthening the coercivity of $\tilde{a}$, it enables to prove that $\Phi^{n}$ is a contraction (for a time step sufficiently small). In the static case, without the help of the inertial term, this fixed-point proof works only for a certain range of physical parameters, for instance when the Young modulus is large compared with the friction coefficient [17, Theorem 11.4].

To conclude this part, we formulate the energy balance. We define the energy at time $t^{n}$ as

$$
E^{n}:=\frac{1}{2}\left(A u^{n}, u^{n}\right)+\frac{1}{2}\left(M^{*} v^{n}, v^{n}\right) .
$$

At each time $t^{n}$, there exist $\lambda_{\mathrm{c}}^{n} \in \partial I_{K}\left(u_{\mathrm{c}}^{n}\right)$ and $\lambda_{\mathrm{f}}^{n} \in \partial_{2} j\left(u^{n}, v_{\mathrm{f}}^{n}\right)$ such that

$$
M^{*} \mathrm{a}_{*}^{n}+A u^{n}+\lambda_{\mathrm{c}}^{n}+\lambda_{\mathrm{f}}^{n}=L\left(t^{n}\right) .
$$

Proceeding as in [18], it is readily shown that

$$
\begin{aligned}
E^{n+1}-E^{n}= & -\frac{1}{2}\left(\lambda_{\mathrm{c}}^{n}+\lambda_{\mathrm{c}}^{n+1}, u^{n+1}-u^{n}\right)-\frac{1}{2}\left(\lambda_{\mathrm{f}}^{n}+\lambda_{\mathrm{f}}^{n+1}, u^{n+1}-u^{n}\right) \\
& +\frac{1}{2}\left(L^{n}+L^{n+1}, u^{n+1}-u^{n}\right) .
\end{aligned}
$$




\section{Convergence of the fully discrete solutions}

We fix the space discretization and we build the approximate solutions $\omega^{\Delta t}:[0, T] \rightarrow V$ as follows:

$$
\begin{aligned}
& \omega^{\Delta t}(t):=u^{n}+v_{*}^{n}\left(t-t^{n}\right)+\frac{1}{2} a_{*}^{n+\frac{1}{2}}\left(t-t^{n}\right)^{2} \quad \forall t \in\left[t^{n}, t^{n+1}\right), \\
& \omega^{\Delta t}(T):=u^{N} .
\end{aligned}
$$

It is readily verified that, by construction, $\omega^{\Delta t} \in C^{0}([0, T] ; V)$ and $\omega_{*}^{\Delta t} \in C^{1}\left([0, T] ; V^{*}\right)$. Furthermore, $\omega^{\Delta t} \in W^{1, \infty}(0, T ; V)$. We are now going to prove the convergence of these approximate solutions to the semi-discrete solution $u$ of Problem 1 . In this section, the notation $A \lesssim B$ means that $A \leq c B$ with a constant $c$ independent of $\Delta t$, but which can depend on $h$. We assume without loss of generality that $\Delta t \leq 1$.

Lemma 4 Let $\left(u^{n}, v^{n}, a^{n}\right)$ solve, for all $n \in\{0, \ldots, N\}$, Problem 2. Then, for $\Delta t$ small enough,

$$
\left\|u^{n}\right\| \lesssim 1, \quad\left\|v_{*}^{n}\right\| \lesssim 1, \quad\left\|a_{*}^{n}\right\| \lesssim 1
$$

Proof (i) Let $n \in\{0, \ldots, N\}$. From (50) we deduce $A^{\mathrm{c}} u^{n}+\lambda_{\mathrm{c}}^{n}=L^{\mathrm{c}}\left(t^{n}\right)$, and then, $\left\|\lambda_{\mathrm{c}}^{n}\right\| \lesssim\left\|u^{n}\right\|+\left\|L\left(t^{n}\right)\right\|$. Owing to the inequality (16), we obtain $\left\|\lambda_{\mathrm{f}}^{n}\right\| \lesssim\left\|u^{n}\right\|$. Hence, owing to the equilibrium equation (50), $\left\|a_{*}^{n}\right\| \lesssim\left\|u^{n}\right\|+\left\|L\left(t^{n}\right)\right\|$.

(ii) Using the energy balance (51), it follows that

$$
E^{n+1}-E^{n} \lesssim\left(\left\|u^{n}\right\|+\left\|u^{n+1}\right\|+\left\|L\left(t^{n}\right)\right\|+\left\|L\left(t^{n+1}\right)\right\|\right)\left\|u^{n+1}-u^{n}\right\| .
$$

Observing that

$$
\begin{aligned}
\left\|u^{n+1}-u^{n}\right\| & \leq\left\|u_{*}^{n+1}-u_{*}^{n}\right\|+\left\|q^{n+1}\left(u_{*}^{n+1}\right)-q^{n+1}\left(u_{*}^{n}\right)\right\|+\left\|q^{n+1}\left(u_{*}^{n}\right)-q^{n}\left(u_{*}^{n}\right)\right\| \\
& \lesssim\left\|u_{*}^{n+1}-u_{*}^{n}\right\|+\left\|L\left(t^{n+1}\right)-L\left(t^{n}\right)\right\| \\
& \lesssim\left\|u_{*}^{n+1}-u_{*}^{n}\right\|+\left\|L\left(t^{n+1}\right)\right\|+\left\|L\left(t^{n}\right)\right\|,
\end{aligned}
$$

we infer

$$
\begin{aligned}
E^{n+1}-E^{n} \lesssim\left(\left\|u^{n}\right\|+\left\|u^{n+1}\right\|+\left\|L\left(t^{n}\right)\right\|+\left\|L\left(t^{n+1}\right)\right\|\right) \\
\left(\left\|u_{*}^{n+1}-u_{*}^{n}\right\|+\left\|L\left(t^{n+1}\right)\right\|+\left\|L\left(t^{n}\right)\right\|\right) .
\end{aligned}
$$

Using (38),

$$
\begin{array}{r}
E^{n+1}-E^{n} \lesssim \Delta t\left(\left\|u^{n}\right\|+\left\|u^{n+1}\right\|+\left\|L\left(t^{n}\right)\right\|+\left\|L\left(t^{n+1}\right)\right\|\right) \\
\left(\left\|v_{*}^{n}\right\|+\frac{\Delta t}{2}\left\|a_{*}^{n+\frac{1}{2}}\right\|+\left\|L\left(t^{n+1}\right)\right\|+\left\|L\left(t^{n}\right)\right\|\right) .
\end{array}
$$

Thus, using the previous bound on $\left\|a_{*}^{n}\right\|$ and $\left\|a_{*}^{n+1}\right\|$, and since $\Delta t \leq 1$,

$$
\begin{aligned}
E^{n+1}-E^{n} \lesssim \Delta t\left(\left\|u^{n}\right\|+\right. & \left.\left\|u^{n+1}\right\|+\left\|L\left(t^{n}\right)\right\|+\left\|L\left(t^{n+1}\right)\right\|\right) \\
& \left(\left\|v_{*}^{n}\right\|+\left\|u^{n}\right\|+\left\|u^{n+1}\right\|+\left\|L\left(t^{n+1}\right)\right\|+\left\|L\left(t^{n}\right)\right\|\right) .
\end{aligned}
$$


Now, using Young's inequality and the coercivity of the energy $E^{n}$,

$$
E^{n+1}-E^{n} \leq C_{1} \Delta t E^{n+1}+C_{2} \Delta t E^{n}+C_{3} \Delta t\left(\left\|L\left(t^{n}\right)\right\|^{2}+\left\|L\left(t^{n+1}\right)\right\|^{2}\right),
$$

where $C_{1}, C_{2}, C_{3}$ are three constants independent of $\Delta t$. Next,

$E^{n+1}-E^{n} \leq C_{1} \Delta t\left(E^{n+1}-E^{n}\right)+\left(C_{1}+C_{2}\right) \Delta t E^{n}+C_{3} \Delta t\left(\left\|L\left(t^{n}\right)\right\|^{2}+\left\|L\left(t^{n+1}\right)\right\|^{2}\right)$,

For $\Delta t \leq 1 /\left(2 C_{1}\right)$,

$$
\frac{1}{2}\left(E^{n+1}-E^{n}\right) \leq\left(C_{1}+C_{2}\right) \Delta t E^{n}+C_{3} \Delta t\left(\left\|L\left(t^{n}\right)\right\|^{2}+\left\|L\left(t^{n+1}\right)\right\|^{2}\right),
$$

so that

$$
E^{n+1}-E^{n} \lesssim \Delta t\left(E^{n}+\left\|L\left(t^{n}\right)\right\|^{2}+\left\|L\left(t^{n+1}\right)\right\|^{2}\right) .
$$

Finally, using a discrete Gronwall lemma,

$$
E^{n} \lesssim E^{0}+\sum_{j=0}^{n} \Delta t\left\|L\left(t^{j}\right)\right\|^{2} \lesssim 1 .
$$

Then, it is straightforward to obtain the estimates (54).

Theorem 5 The following convergence results hold true as $\Delta t \rightarrow 0$,

$$
\begin{aligned}
& \omega^{\Delta t} \rightarrow u \quad \text { in } C^{0}([0, T] ; V), \\
& \dot{\omega}_{*}^{\Delta t} \rightarrow \dot{u}_{*} \text { in } C^{0}\left([0, T] ; V^{*}\right), \\
& \ddot{\omega}_{*}^{\Delta t} \rightarrow \ddot{u}_{*} \text { weakly } * \text { in } L^{\infty}\left(0, T ; V^{*}\right),
\end{aligned}
$$

where u solves Problem 1.

Proof (i) From the estimates (54), we deduce that

$$
\begin{aligned}
\left\|\omega^{\Delta t}\right\|_{L^{\infty}(0, T ; V)} & \lesssim 1, \quad\left\|\dot{\omega}^{\Delta t}\right\|_{L^{\infty}(0, T ; V)} \lesssim 1, \\
\left\|\dot{\omega}_{*}^{\Delta t}\right\|_{L^{\infty}\left(0, T ; V^{*}\right)} & \lesssim 1, \quad\left\|\ddot{\omega}_{*}^{\Delta t}\right\|_{L^{\infty}\left(0, T ; V^{*}\right)} \lesssim 1 .
\end{aligned}
$$

(ii) Using standard compactness arguments [22], there exists $\omega \in C^{0}(0, T ; V)$ such that $\dot{\omega}_{*} \in C^{0}\left(0, T ; V^{*}\right), \ddot{\omega}_{*} \in L^{\infty}\left(0, T ; V^{*}\right)$, and, up to a subsequence,

$$
\begin{aligned}
& \omega^{\Delta t} \rightarrow \omega \text { in } C^{0}([0, T] ; V), \\
& \dot{\omega}_{*}^{\Delta t} \rightarrow \dot{\omega}_{*} \text { in } C^{0}\left([0, T] ; V^{*}\right), \\
& \ddot{\omega}_{*}^{\Delta t} \rightarrow \ddot{\omega}_{*} \text { weakly } * \text { in } L^{\infty}\left(0, T ; V^{*}\right) .
\end{aligned}
$$

(iii) Next, we introduce the auxiliary (piecewise constant in time) approximate solutions $\underline{\omega}^{\Delta t}:[0, T] \rightarrow V$ and $\zeta_{*}^{\Delta t}:[0, T] \rightarrow V^{*}$ such that

$$
\begin{array}{lll}
\zeta_{*}^{\Delta t}(t):=v_{*}^{n+1} & \forall t \in\left[t^{n}, t^{n+1}\right), & \zeta_{*}^{\Delta t}(T):=v_{*}^{N}, \\
\underline{\omega}^{\Delta t}(t):=u^{n+1} & \forall t \in\left[t^{n}, t^{n+1}\right), & \underline{\omega}^{\Delta t}(T):=u^{N} .
\end{array}
$$


By definition of the approximate solutions $\omega^{\Delta t}$ and $\underline{\omega}^{\Delta t}$, and using relation (38), $\forall n \in\{0, \ldots, N\}, \forall t \in\left[t^{n}, t^{n+1}\right)$,

$$
\begin{aligned}
\left\|\omega^{\Delta t}(t)-\underline{\omega}^{\Delta t}(t)\right\| & \leq\left\|u^{n+1}-u^{n}\right\|+\Delta t\left\|v_{*}^{n}\right\|+\frac{1}{2} \Delta t^{2}\left\|a_{*}^{n+\frac{1}{2}}\right\| \\
& \leq 2 \Delta t\left\|v_{*}^{n}\right\|+\Delta t^{2}\left\|a_{*}^{n+\frac{1}{2}}\right\| .
\end{aligned}
$$

Hence, using estimates (54),

$$
\left\|\omega^{\Delta t}(t)-\underline{\omega}^{\Delta t}(t)\right\| \lesssim \Delta t \quad \text { a.e. in }[0, T] .
$$

We deduce that $\underline{\omega}^{\Delta t} \rightarrow \omega$ in $L^{\infty}(0, T ; V)$. In the same way, we prove that $\zeta_{*}^{\Delta t} \rightarrow \dot{\omega}_{*}$ in $L^{\infty}\left(0, T ; V^{*}\right)$. We define an approximate external force vector,

$$
\underline{L}^{\Delta t}(t):=L\left(t^{n+1}\right) \quad \forall t \in\left[t^{n}, t^{n+1}\right), \quad \underline{L}^{\Delta t}(T):=L\left(t^{N}\right) .
$$

Since $t \mapsto L(t)$ is Lipschitz continuous, $\underline{L}^{\Delta t} \rightarrow L$ in $L^{\infty}(0, T ; V)$.

(iv) Owing to (36), the approximate solutions satisfy

$$
M^{*} \ddot{\omega}_{*}^{\Delta t} \in-A^{*} \underline{\omega}^{\Delta t}-\partial_{2} j\left(\underline{\omega}^{\Delta t}, \zeta_{\mathrm{f}}^{\Delta t}\right)+\underline{L}^{\Delta t *}(t) \quad \text { a.e. in }[0, T],
$$

so that

$$
\begin{aligned}
m^{*}\left(\ddot{\omega}_{*}^{\Delta t}, v_{*}-\zeta_{*}^{\Delta t}\right)+a\left(\underline{\omega}^{\Delta t}, v_{*}-\zeta_{*}^{\Delta t}\right)+j\left(\underline{\omega}^{\Delta t}, v_{\mathrm{f}}\right)-j\left(\underline{\omega}^{\Delta t}, \zeta_{\mathrm{f}}^{\Delta t}\right) \\
\geq\left(\underline{L}^{\Delta t}, v_{*}-\zeta_{*}^{\Delta t}\right) \quad \forall v_{*} \in V^{*}, \text { a.e. in }[0, T] .
\end{aligned}
$$

Passing to the limit,

$$
\begin{aligned}
m^{*}\left(\ddot{\omega}_{*}, v_{*}-\dot{\omega}_{*}\right)+a\left(\omega, v_{*}-\dot{\omega}_{*}\right)+j\left(\omega, v_{\mathrm{f}}\right)-j\left(\omega, \dot{\omega}_{\mathrm{f}}\right) & \\
& \geq l\left(t, v_{*}-\dot{\omega}_{*}\right) \quad \forall v_{*} \in V^{*}, \text { a.e. in }[0, T],
\end{aligned}
$$

and hence

$$
M^{*} \ddot{\omega}_{*} \in-A^{*} \omega-\partial_{2} j\left(\omega, \dot{\omega}_{\mathrm{f}}\right)+L^{*}(t) \quad \text { a.e. in }[0, T] .
$$

By uniqueness of the solution, we conclude that $\omega=u$. This uniqueness also implies that the whole sequence $\left(\omega^{\Delta t}\right)$ converges, not only a subsequence.

\section{References}

1. J.-P. Aubin and H. Frankowska. Set-valued analysis, volume 2 of Systems \& Control: Foundations 83 Applications. Birkhäuser Boston Inc., Boston, MA, 1990.

2. P. Ballard and S. Basseville. Existence and uniqueness for dynamical unilateral contact with Coulomb friction: a model problem. M2AN Math. Model. Numer. Anal., 39(1):59-77, 2005.

3. B. Brogliato. Nonsmooth impact mechanics, volume 220 of Lecture Notes in Control and Information Sciences. Springer-Verlag London Ltd., London, 1996. Models, dynamics and control.

4. F. H. Clarke. Optimization and nonsmooth analysis, volume 5 of Classics in Applied Mathematics. Society for Industrial and Applied Mathematics (SIAM), Philadelphia, PA, second edition, 1990 .

5. M. Cocou. Existence of solutions of a dynamic Signorini's problem with nonlocal friction in viscoelasticity. Z. Angew. Math. Phys., 53(6):1099-1109, 2002. Dedicated to Eugen Soós. 
6. K. Deimling. Multivalued differential equations, volume 1 of de Gruyter Series in Nonlinear Analysis and Applications. Walter de Gruyter \& Co., Berlin, 1992.

7. D. Doyen and A. Ern. Convergence of a space semi-discrete modified mass method for the dynamic Signorini problem. Commun. Math. Sci., 7(4):1063-1072, 2009.

8. D. Doyen, A. Ern, and S. Piperno. Time-integration schemes for the finite element dynamic Signorini problem. submitted.

9. A. Ern and J.-L. Guermond. Theory and Practice of Finite Elements, volume 159 of Applied Mathematical Sciences. Springer-Verlag, New York, 2004.

10. A. F. Filippov. Differential equations with discontinuous righthand sides, volume 18 of Mathematics and its Applications (Soviet Series). Kluwer Academic Publishers Group, Dordrecht, 1988. Translated from the Russian.

11. C. Hager, S. Hüeber, and B. I. Wohlmuth. A stable energy-conserving approach for frictional contact problems based on quadrature formulas. Internat. J. Numer. Methods Engrg., 73(2):205-225, 2008.

12. C. Hager and B. I. Wohlmuth. Analysis of a space-time discretization for dynamic elasticity problems based on mass-free surface elements. SIAM J. Numer. Anal., 47(3):1863-1885, 2009.

13. W. Han and M. Sofonea. Quasistatic contact problems in viscoelasticity and viscoplasticity, volume 30 of AMS/IP Studies in Advanced Mathematics. American Mathematical Society, Providence, RI, 2002.

14. P. Hild and Y. Renard. Local uniqueness and continuation of solutions for the discrete Coulomb friction problem in elastostatics. Quart. Appl. Math., 63(3):553-573, 2005.

15. H. Khenous. Problèmes de contact unilatéral avec frottement de Coulomb en élastostatique et élastodynamique. Etude mathématique et résolution numérique. PhD thesis, INSA de Toulouse, 2005.

16. H. B. Khenous, P. Laborde, and Y. Renard. Mass redistribution method for finite element contact problems in elastodynamics. Eur. J. Mech. A Solids, 27(5):918-932, 2008.

17. N. Kikuchi and J. T. Oden. Contact problems in elasticity: a study of variational inequalities and finite element methods, volume 8 of SIAM Studies in Applied Mathematics. Society for Industrial and Applied Mathematics (SIAM), Philadelphia, PA, 1988.

18. S. Krenk. Energy conservation in Newmark based time integration algorithms. Comput. Methods Appl. Mech. Engrg., 195(44-47):6110-6124, 2006.

19. W. Rudin. Real and complex analysis. McGraw-Hill Book Co., New York, third edition, 1987.

20. G. V. Smirnov. Introduction to the theory of differential inclusions, volume 41 of Graduate Studies in Mathematics. American Mathematical Society, Providence, RI, 2002.

21. D. E. Stewart. Rigid-body dynamics with friction and impact. SIAM Rev., 42(1):3-39 (electronic), 2000

22. R. Temam. Navier-Stokes equations, volume 2 of Studies in Mathematics and its Applications. North-Holland Publishing Co., Amsterdam, third edition, 1984. Theory and numerical analysis, With an appendix by F. Thomasset.

23. T. Warburton and J. S. Hesthaven. On the constants in $h p$-finite element trace inverse inequalities. Comput. Methods Appl. Mech. Engrg., 192(25):2765-2773, 2003. 